\title{
Performance of symmetric and asymmetric links in wireless networks
}

\author{
Yaser Khamayseh ${ }^{1}$, Rabiah Al-Qudah ${ }^{2}$ \\ ${ }^{1}$ Department of Computer Science, Jordan University of Science and Technology, Irbid, Jordan \\ ${ }^{2}$ Department of Computer Science, Concordia University, Montreal, Canada
}

\begin{tabular}{l} 
Article Info \\
\hline Article history: \\
Received Mar 31, 2021 \\
Revised Jun 15, 2021 \\
Accepted Aug 4, 2021 \\
\hline
\end{tabular}

Keywords:

Asymmetric

Capacity

Radii

Resource allocation

Sensing

Symmetric

Wireless networks

\begin{abstract}
Wireless networks are designed to provide the enabling infrastructure for emerging technological advancements. The main characteristics of wireless networks are: Mobility, power constraints, high packet loss, and lower bandwidth. Nodes' mobility is a crucial consideration for wireless networks, as nodes are moving all the time, and this may result in loss of connectivity in the network. The goal of this work is to explore the effect of replacing the generally held assumption of symmetric radii for wireless networks with asymmetric radii. This replacement may have a direct impact on the connectivity, throughput, and collision avoidance mechanism of mobile networks. The proposed replacement may also impact other mobile protocol's functionality. In this work, we are mainly concerned with building and maintaining fully connected wireless network with the asymmetric assumption. For this extent, we propose to study the effect of the asymmetric links assumption on the network performance using extensive simulation experiments. Extensive simulation experiments were performed to measure the impact of these parameters. Finally, a resource allocation scheme for wireless networks is proposed for the dual rate scenario. The performance of the proposed framework is evaluated using simulation.
\end{abstract}

This is an open access article under the CC BY-SA license.

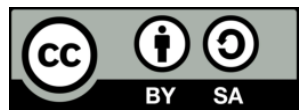

\section{Corresponding Author:}

Yaser Khamayseh

Department of Computer Science, Jordan University of Science and Technology

Irbid, 22110, Jordan

Email: yaser@just.edu.jo

\section{INTRODUCTION}

The vast growth in the deployment and popularity of wireless and mobile networks attracted the attention of researches toward evaluating and enhancing the performance of the mobile and wireless networks' protocols [1], [2]. One development direction adopted by some researchers was to apply and implement the protocols used by wired networks in the wireless domain. While designing any protocol or standard for mobile networks, one must consider several key differences that exist between wireless networks and wired networks [3]. The main characteristics of wireless networks are: i) mobility: although wireless does not constitute mobility by default, however, the main gain of the wireless technologies is to provide mobility to the users. It is unrealistic to design a wireless network that is not mobile. Therefore, in this paper, we assume the two terms are associated with each other; ii) power constraints: devices are typically battery operated, hence power resources are scarce. Moreover, due to health and regulations concerns, devices maximum transmission powers are set to a certain limit; iii) high packet loss: Wireless environments are noisy and suffer from many losses due to various reasons such as fading, attenuations, and multipath propagations; and iv) lower bandwidth: The available bandwidth to the wireless networks is typically lower than the available bandwidth to wired networks such as fiber optics networks. 
Mobility of the network is a crucial part. This is because nodes are constantly moving, and this may result in loss of connectivity in the network. While the node is moving, it is possible that it might lose a link with its neighbor(s) and gain new link(s) with other node(s). These dynamics, and in some cases, fast changes in the network configuration/topology might lead to a disconnected network where one or more nodes cannot access other nodes. And it is possible that in some cases the network will become connected again after a while. In order to carry out transmission and reception between two nodes in a wireless network, the transmitter and receiver must be within the same range. In addition to environmental factors, the power level at the transmitter and receiver stations influences these two ranges, respectively. Each station has a limited power for transmitting and receiving signals. Due to the limitations of wireless medium, mobile networks suffer from high packet loss and lower bandwidth constraints. Several standards have been proposed for wireless networks. For example, the IEEE 802.11 standard is used to describe the physical and medium access control (MAC) layers of WLAN. The functionality of the MAC layer is described by the distributed coordination function (DCF) [3].

A key difference between the wired and the wireless domains is apparent in the collision management policy. The core difference between CSMA/CA (the policy adopted in the wired domain) and the CSMA/CD (the policy adopted in the wireless domain) is that CSMA/CA tries to avoid collisions before transmission by means of collision detection. However, the detection component is not practical in the wireless domain, hence it adopted the collision avoidance policy. In CSMA/CA, each node listens for ongoing transmissions before it starts transmitting. Furthermore, each node is free to transmit when it has data to send. The collision avoidance mechanism is enforced through the RTS/CTS scheme. The RTS/CTS mechanism is a simple and easy to implement protocol, however, it misses on many possible non-interfering concurrent transmissions between other nodes in the network. Due to power constraints, each node in a mobile network has a limited transmitting and sensing (receiving) radius. The transmission radius is the range in which the node can send messages to another node within this range. On the other hand, the sensing radius is the range within which a node can sense (hear) transmission from other nodes. Most research done for mobile networks assumes that the transmitting and the sensing range for each station are the same. But what if they are not?.

The first question to address is whether a station can have different transmitting and sensing radii?. The answer is yes. In some cases, the station is required to reduce its transmitting power for many reasons. For example, in case of the Bluetooth technology [4], the maximum number of stations allowed per a picocell is 7 stations. If a cell contains more than 7 stations, each station starts reducing its transmitting power in order to limit the cell size to 7. Another example for reduction of transmitting radius is to control the interference level experienced by all nodes so that the network can guarantee a certain level of quality of service (QoS). QoS is highly influenced by the power-noise ratio (i.e., interference level).

Once a node transmits a signal, the radio wave propagates in the air. The signal strength is subject to several limiting factors due to reflection, diffraction, and scattering of the propagated signal [5]. Therefore, the received signal's power by the receiving node is typically lower the transmitted signal's power. The difference between the received power and the transmitted power is referred to as path loss $(P L)$. The most common model for estimating path loss in open spaces (i.e., line of sight) is Friss free space model [5]. In Friss model, the received power $\left(P_{r}\right)$ is estimated as (1):

$$
P_{r}(d)=\frac{P_{t} G_{r} G_{t} \lambda^{2}}{(4 \pi d)^{2} L}
$$

where, $d$ is the distance between the receiver and the transmitter, $P_{t}$ is the power of transmitted signal, $G_{r}$ is the receiver's antenna gain, $G_{t}$ is the transmitter's antenna gain, $\lambda$ is the wavelength, and $L$ is the loss factor. It is used to measure the loss due to hardware imperfections and is typically set to $1 . d$ and $\lambda$ are measured in meters, and $P_{t}, G_{t}$, and $G_{r}$ are measured in db. Now, we can use (1) to compute the path loss [5]:

$$
P L(d b)=10 \log _{10} \frac{P_{r}}{P_{t}}=10 \log _{10} \frac{G_{r} G_{t} \lambda^{2}}{(4 \pi d)^{2} L}
$$

From (1) and (2), the amount of signal degradation depends on several factors: antennas, wavelength (i.e., frequency), and the distance. In top of these factors, there are other factors that may affect the quality of the transmitted signals such as surrounding environment (e.g., rain, and fog), number of objects, and number of active nodes transmitting using the same frequency or even different frequencies. Due to this complex and variable nature of effecting factors, it is hard to predict the path loss, though there exist many empirical and theoretical path loss prediction models such as log shadow model. Even more, it might be unrealistic to assume that both nodes have the same transmission and sensing radii, as each node exhibits slightly different 
conditions. Moreover, predicting or measuring the quality of the wireless signals is even more challenging in extreme surroundings such as wireless underground sensor network (WUSN) [6]. The relationship between distance and transmission power is examined in [7] for wireless mesh network (WMN), in which nodes can be either mobile or stationary. In this work, we examine this relationship in all mobile networks.

The goal of this work is to study the effect of replacing the generally held assumption of symmetric radii for mobile networks with asymmetric radii. This replacement may have a direct impact on the connectivity, throughput and collision avoidance mechanism of mobile networks. The proposed replacement may also impact other mobile protocol's functionality. In this work, we are primarily concerned with connectivity issues with the asymmetric assumption. Finally, a resource allocation mechanism is proposed to provide a fair sharing mechanism among all active nodes. In this mechanism, the resources allocation problem is formulated as a scheduling problem, then, a heuristic dual-rate algorithm is proposed to maximize the system's throughput while serving a maximal number of users.

The rest of this paper is organized as follows: Section 2 highlights the main findings in the literature. Section 3 presents the study methodology in evaluating the effect of the symmetric assumption. Section 4 presents the simulation setup. The results are discussed in section 5. Dual-rate resource allocation framework is presented in section 6 along with some preliminary results. Finally, the paper is concluded in section 7.

\section{LITERATURE SURVEY}

Symmetric and asymmetric transmission systems were assumed in [8]. The main goal of the work in [8] is to have "good" routes from all nodes to the sink node. The authors try to use any symmetric and/or asymmetric links between nodes to construct routing tree. The motivation of their work is that many previous good algorithms only use the symmetric links in constructing the routing tree, however, the real wireless network contains many asymmetric links and avoiding them in constructing the routing tree would miss many possible good routes. Moreover, the main goal in wireless sensor network is to have one direction routes to pass the packets to the sink nodes, thus the assumption of using asymmetric links is valid. This approach was named reliable collection protocol for aggregating data packets (RECPE). The performance of the RECPE approach was tested using the TOSSIM simulator. The obtained results show that the RECPE approach achieved substantial performance gain in terms of latency, delivery cost, and packet loss ratio against the collection tree protocol that uses expected transmission count (ETX) as the routing metric. RECPE achieves better performance when more links are asymmetric as motivated by the approach.

The testbed deployed in [9] found that substantial percentage of links in wireless sensor network is asymmetric and many of them are even unidirectional. The authors proposed a new one-way link metric expected number of transmissions over forward links (ETF). The authors compared ETF against expected transmission count in high fidelity testbed (on different platforms) and showed that exploiting asymmetric links (via ETF) significantly improves the performance. The authors suggest that their approach can be further used in other wireless environments rather than just WSNs such as wireless mesh networks in general as the assumptions used in their derivation holds.

Symmetric wireless transmission links were assumed in [10]. The authors study the critical transmission radius in wireless ad-hoc networks where k-connectivity topology is assumed. The goal was to provide asymptotic distribution of the critical transmission radius in such topologies. In addition, the authors provide a critical neighbor number upper bound estimation. The results can be used in developing different protocols and algorithms in wireless networks where the topology assumptions hold. A symmetric transmission range was assumed and studied in [11] to develop estimation for the best number of cluster heads in wireless sensor networks. The authors determined the number of relay nodes and the energy consumption in the Intra- and Inter-cluster operations. By quantifying the energy consumption, the authors determine the optimal size of a local cluster for a given number of cluster heads which aids to determine the optimal number of cluster heads.

Raza et al. [12] proposed a relay selection scheme for cooperative wireless networks based on both link's weight and link's quality. The proposed scheme considered both symmetric and asymmetric links. While the work in [13] sheds the light on the importance of differentiating between the communication in 2 directions (the asymmetric assumption) and how it may affect the network performance. The work in [14] investigated the effect of common assumptions (axioms) used by researchers in the ad-hoc networks simulation studies. It questioned the validity of these assumptions and its relevance to the real world. It examined the following key axioms: the world is flat, a radio's transmission area is circular, all radios have equal range, if I can hear you, and you can hear me (symmetry), if I can hear you at all, I can hear you perfectly, and signal strength is a simple function of distance. The authors challenged all these assumption in the real world and proved that they don't hold all the time and there is a need to examine the effect of these assumptions on the performance of ad-hoc networks as well as develop new tools to account for these findings. 
Salahat et al. in [15] discussed the asymmetric assumption in wireless networks and the main causes of the asymmetry in wireless channels. Using empirical study, the authors proved the importance of the asymmetric assumption for both line of sight (LOS) and none line of sight (NLOS) environments. The symmetric assumption was also investigated in [16] for wireless underground sensor networks (WUSN) that use soil as the communication medium, empirical results revealed that the asymmetric assumption holds for the cases of aboveground-to-underground links and for the underground-to-underground links.

Additionally, many studies have been conducted to investigate the optimal value for the transmitting radius of a mobile node. Theoretically, it has been established that in order to achieve a good performance in a mobile environment, the transmitting radius should cover a region with 6 neighbors [17], [18]. The choice of an optimal transmitting radius is affected by several objectives that must also be achieved. The objectives to be met are as follows: Minimizing interference level, minimizing delay in the network, maximizing throughput, utilizing the available power level efficiently, and increasing connectivity

A tradeoff must be achieved between the optimal transmitting radius and these objectives. Thus, the value of the transmitting radius can be defined as a function that considers all these factors. To our knowledge, most of the works in the literature considered the potential benefits/limitations of the asymmetric links and designed protocols to exploit these characteristics, no work was done to fully investigate its effect on the network performance as proposed in this work. In this work, we are concerned with the assumption of asymmetric links that may affect mobile network connectivity.

The resource allocation problem in wireless domain is investigated on [19], in which the authors proposed a 2-stage linear probabilistic solution for a network powered by and edge computing unit. The authored derived a sub-optimal sampling solution as the original problem is a continuous one. The proposed solution managed to increase the system throughput by distributing the available resources (i.e., bandwidth, power and channels) among the users. Recently, the use of advanced artificial intelligence approaches (such as deep reinforcement learning) to tackle the resource allocation problem in the wireless domain is apparent [20], [21]. However, these techniques require substantial computations and may not be suitable for all wireless networks' applications/types.

The buffer link capacity issues and challenges are discussed in [22], the work in [22] identifies the main issues related to buffer size in wireless networks and how it can impact the performance of the network, it identifies link scheduling, adaptive link rates, frame aggregation, variable packet inter-service time, and multihopping as the key challenges for buffer management in wireless domains. The work then suggests some possible alternative to overcome these challenges: single hope solutions, multi-hope solutions and Active queue management. Our work, provide an extensive simulation experiments to highlight the impact of buffer size and capacity on the performance of the wireless networks.

\section{METHOD}

\subsection{Symmetric vs asymmetric system model}

To investigate the effect of the symmetric assumption on the network performance and connectivity, we conducted extensive simulation experiments under various scenarios. An event-based simulator was built using $\mathrm{C}++$ to study the effect of asymmetric links over network connectivity. It simulates a scenario with mobile nodes in a rectangular plane of $1500 \times 300 \mathrm{~m}$, with each node having different transmission/sensing ranges. The mobility model of the nodes in the simulation is based on the 'random waypoint' model [23]. Mobile nodes are located at random coordinates in the rectangular area. According to the 'random waypoint' model, nodes are initially in the pause state. The pause time for each node is generated based on an exponential distribution function with various means. Once the pause time for a node has expired, the node is picked for movement to a new randomly generated location. The node moves towards its destination with a speed generated based on a uniform distribution. On reaching the destination, the nodes pause again and repeats the above procedure till the end of the simulation.

During movement, a node can enter the transmitting or receiving radii of other nodes. At this time, a connection is established between the concerned nodes. Similarly, nodes that previously had formed links with other nodes may now leave the transmitting/receiving radii of these nodes resulting in a loss of connection. Figure 1 shows a communication scenario between two nodes with different transmission and sensing radii.

In the case of an add link or remove link event, a graph connectivity check is performed using the breadth first search (BFS) algorithm [24]. The number of neighbors for each node is also calculated (this information is used later to compute the average number of neighbors). The following parameters and factors were assumed in our simulation:

- Rectangular area of $1500 \mathrm{~m}$ by $300 \mathrm{~m}$ for the random waypoint mobility model

- The speed of the nodes is generated based on a uniform distribution in the range $2 \mathrm{~m} / \mathrm{s}$ and $20 \mathrm{~m} / \mathrm{s}$ 
- The total simulation time is set at 1,000,000 seconds

- Number of nodes is chosen from 5, 10, 20 or 50

- All mobile nodes pause for a time based on an exponential distribution with means 0,10 and 20 seconds

- The transmitting and sensing radii were varied for three different sets of experiments as shown in Table 1 .

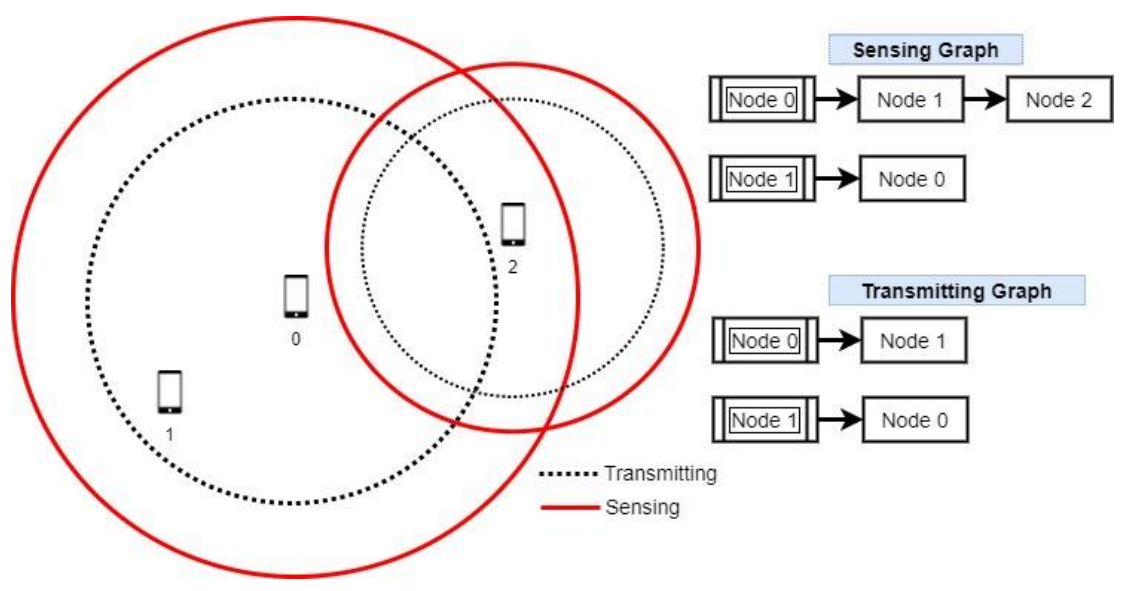

Figure 1. Representation of the transmitting and sensing graphs under asymmetric links

Table 1. Sets of radii for different experiments

\begin{tabular}{ccc}
\hline & Transmission radius & Sensing radius \\
\hline Symmetric & $\{20,50,80,150,300,500\}$ & $\{20,50,80,150,300,500\}$ \\
Symmetric with mean & $\{20,50,80,150,300,500\}$ & $\{20,50,80,150,300,500\}$ \\
Asymmetric & $\{20,50,80,150,300,500\}$ & $\{100,200,300,400,500\}$ \\
\hline
\end{tabular}

This paper examines the effect of asymmetric links over connectivity. One of the performance metrics used to evaluate this effect is the probability of the network connectivity. The second metric of concern is the average number of nodes in the neighborhood of each mobile node's sensing radius. This can be viewed as the cost for maintaining the same level of connectivity. In the case of the first metric, we are trying to measure how long the network stays connected. The probability of connectivity is the time of the network being connected over the total simulation run time. Traditionally, researchers have held the view that nodes have symmetric radii (i.e., similar transmission and sensing radii). However, mobile nodes can have different radii. In this case, we expect that connectivity of the network is not affected since connectivity is primarily established due to the transmitting radius even though the definition of connectivity says that a network is connected if a node can send and receive from all other nodes (sensing radius is larger than transmitting radius). If a node can sense other nodes but cannot transmit to them, the graph is not connected by default. As a result, increasing the sensing radius (asymmetric case), will not affect connectivity.

$$
P_{\text {connectivity }}=\frac{\text { Connected Time }}{\text { Total Time }} \times 100 \%
$$

The second metric is described by how many nodes the focal node can sense on average. As described in the last paragraph, we expect that connectivity is not affected due to increasing sensing radius. However, increased sensing radius will increase the number of nodes a particular node can sense. This may also affect the collision avoidance mechanism, for example the RTS/CTS method. For node $i$,

$$
A v g_{\text {Nodes }}^{i}=\frac{\sum \text { number of neighbours }}{n}
$$

In the asymmetric case, we expect to keep the same level of connectivity but expect the performance or efficiency of individual nodes to degrade. With increased sensing radius in the asymmetric case, the cost endured by each node to maintain the same level of connectivity becomes larger due to the number of nodes being sensed. In this case, individual nodes have to compete with a larger number of nodes for transmission. In general, the system throughput will remain the same. For verification purposes, several tests were performed to ensure that the simulation model is correctly implemented. The simulation model was 
implemented using a top-down modular design approach. Antibugging was applied to test the program for errors by inserting additional checks and outputs in the program. The graph data structures were analyzed to ensure that the correct entries were being made. Similarly, the heap was traced for proper event execution. A trace was carried out with a list of a time ordered events and associated variables. In particular, the movement of nodes was traced through the system. A timeline was prepared manually and the nodes behavior in the system was compared against this timeline. Several input parameters were tested as a part of the continuity test. A degeneracy test was done to verify the implementation of the model by providing extreme values for workload parameters.

\section{SYMMETRIC VS ASYMMETRIC LINKS RESULTS AND DISCUSSION}

This section presents and discusses the obtained results. In this scenario, both the transmitting and sensing radii have the same values for all nodes. The symmetric link assumption is then extended with the transmitting radius being generated based on a mean. Here, the mean of the transmitting radius is the same as the sensing radius. All nodes thus have the same sensing radii but have different transmitting radii. Finally, we also present the results for the asymmetric link case. All nodes have the same sensing radii but have different transmitting radii. Furthermore, the transmitting radii are different from the sensing radii.

\subsection{Symmetric link results}

Figure 2 depicts the effect of increasing the number of mobile nodes over average number of nodes and connectivity for the symmetric link assumption. Figure 2(a) depicts the average number of nodes for mean pause time 0 and number of nodes 5, 10, 20 and 50. Figure 2(b) depicts the same results for the probability of connectivity. Both the graphs show results for the symmetric case with various radii values $(20,50,80,150,300$ and 500, same for transmission and sensing). By default, with an increase in the transmitting/sensing radii, the average number of nodes for a particular node will increase. Similarly, with an increase in the total number of nodes in the mobile plane, the average number of nodes for a node also increases. At a transmitting/sensing radius of $300 \mathrm{~m}$, the average number of nodes seems to stabilize.

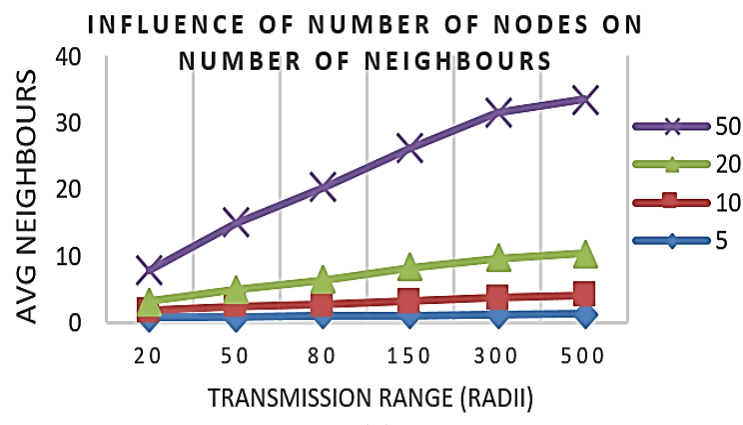

(a)

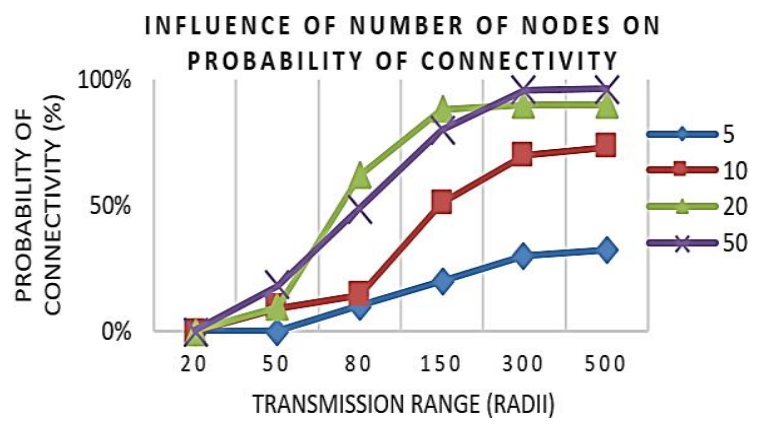

(b)

Figure 2. Effect of increasing number of nodes over: (a) average number of nodes, (b) probability of connectivity in the symmetric case

Furthermore, with an increase in the number of nodes in the symmetric case, we can observe that the connectivity also increases. In the case of 50 nodes, the connectivity reaches $99 \%$. Furthermore, with the increase in transmission radius (symmetric), the probability of connectivity also increases. When the transmission radius is in the range of approximately 50 to 150 meters, the connectivity increases significantly $(\sim 20 \%$ to $\sim 90 \%)$. Below a transmitting radius of 50 meters, the coverage for each node is small and fewer nodes can communicate with each other resulting in low connectivity. After 150 meters, there is no significant improvement in connectivity as a path to every other node can be easily established. This behavior is clearer in the case of a larger number of nodes (e.g., 20 and 50 nodes). These observations were recorded for pause time of 0 seconds. The same behavior was noted for pause times of 10 and 20 seconds.

\subsection{Extended symmetric link results}

Figure 3 presents the effect of increasing number of mobile nodes over average number of nodes and connectivity for the extended symmetric link assumption. Figure 3(a) depicts the average number of nodes for mean pause time 0 and number of nodes 5, 10, 20 and 50. Figure 3(b) depicts the same results for the 
probability of connectivity. Both the graphs show results for the extended symmetric case with various radii values (20,50,80, 150, 300 and 500, same for transmission and sensing). The results for the extended symmetric case are identical to the symmetric case. A simple comparison between Figures 2 and 3 shows that there are slightly minimal differences between the results for the symmetric and the extended symmetric cases.

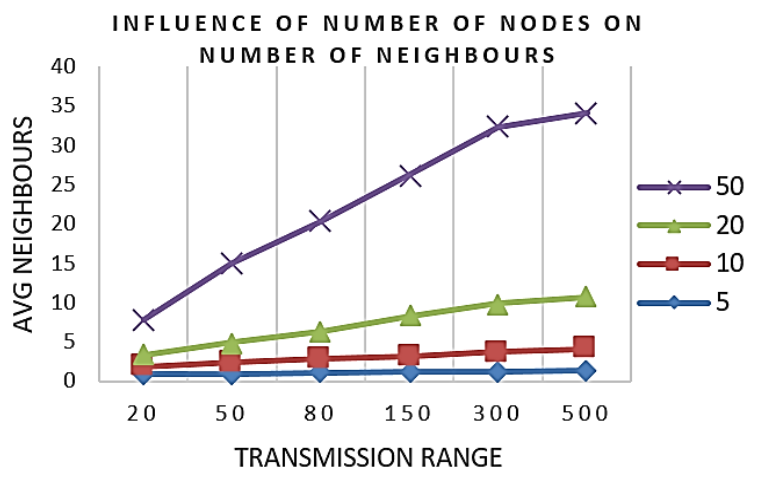

(a)

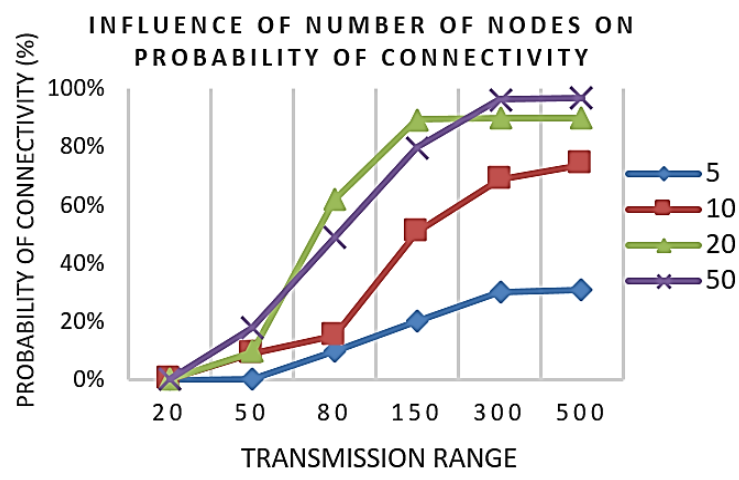

(b)

Figure 3. Effect of increasing number of nodes over: (a) average number of nodes, (b) probability of connectivity in the extended symmetric case

\subsection{Asymmetric link results}

Figure 4 presents the effect of increasing number of mobile nodes over connectivity for the asymmetric case. The graph shows three sets of lines for different number of nodes (first set of lines for 5 nodes, second set of lines for 10 nodes and last set of lines for 20 nodes). All graphs are for pause time of 0. The transmission radius is different from the sensing radius for each node. Traditionally, the sensing radius is larger than the transmission radius. Figure 4 however shows certain scenarios where the transmission radius is larger (e.g., transmission radius $=500$ and sensing radius $=100$ ). Figure 4 shows the results for the asymmetric link assumption. In particular, Figure 4 describes the effect of increasing number of nodes over the probability of connectivity in the network. Three major conclusions can be drawn from Figure 4:

- An increasing number of nodes lead to increased connectivity

- Increased transmitting radius also results in increased connectivity

- Increased sensing radius does not have a significant impact on connectivity.

It also has cases where the transmitting radius is larger than the sensing radius. In these scenarios, the behavior of connectivity is similar to the traditional approach. With 20 nodes and a transmitting radius between $50 \mathrm{~m}$ and $150 \mathrm{~m}$, there is a significant increase in connectivity. Above $150 \mathrm{~m}$ connectivity stabilizes. The reasons for this have been discussed already. Figure 5 shows the effect of increasing number of mobile nodes over average number of neighbors for the asymmetric case. The graph is split into three column graphs. (First set of columns for 5 nodes, second set of columns for 10 nodes and last set of columns for 20 nodes). All graphs are for pause time 0 . The transmission radius is different from the sensing radius for each node. Figure 5 shows certain scenarios where the transmission radius is larger than the sensing radius (e.g., transmission radius $=500$ and sensing radius $=100$ ). Figure 5 shows the effect of increasing number of nodes over the average number of neighbors for each node in the asymmetric case. Three major conclusions can also be drawn from Figure 5:

- Increasing number of nodes lead to increased average neighbors

- Increased sensing radius results in increased average neighbors

- Increased transmitting radius has a tiny impact and causes average neighbors to go down.

At the start of the simulation, we predicted that the transmitting radius would have no impact on average neighbors however the results show a tiny decrease with increasing transmitting radius. Figure 6 presents the effect of increasing sensing radius over the probability of connectivity for the asymmetric case. The pause time is set at 0 and the number of nodes is 20 . Based on the results from Figure 6 , the sensing radius does not influence the connectivity. Figure 7 shows the probability of connectivity in the symmetric, extended symmetric and asymmetric case with pause time 0 . The number of nodes is chosen to be 20 . The effect of increasing sensing radius over the probability of connectivity for the asymmetric case is shown in Figure 6. This Figure supports our observation that sensing radius has a minimal impact on connectivity. 


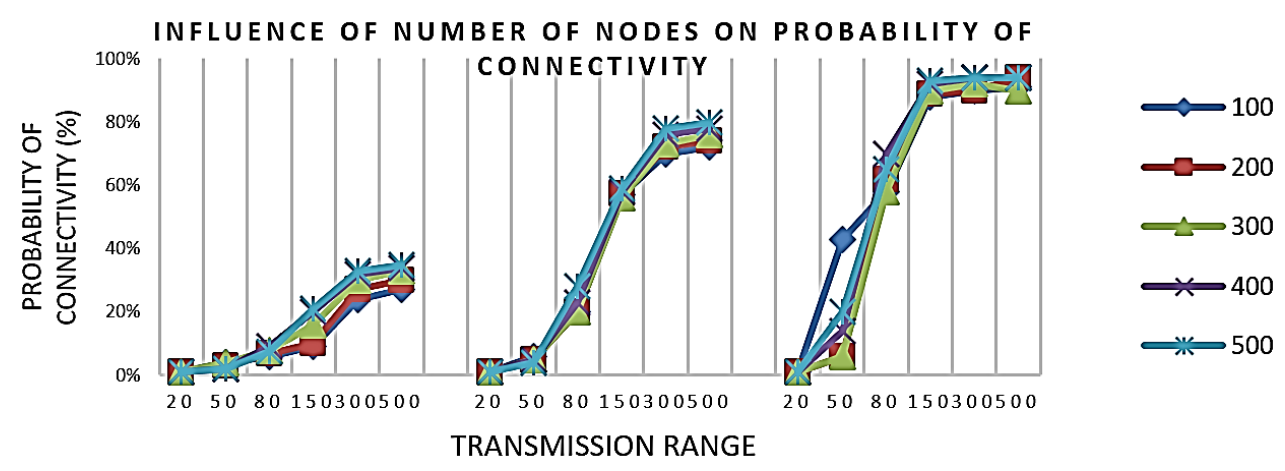

Figure 4. Effect of increasing number of nodes over the probability of connectivity-asymmetric case

INFLUENCE OF NUMBER OF NODES ON NUMBER OF NEIGHBOURS

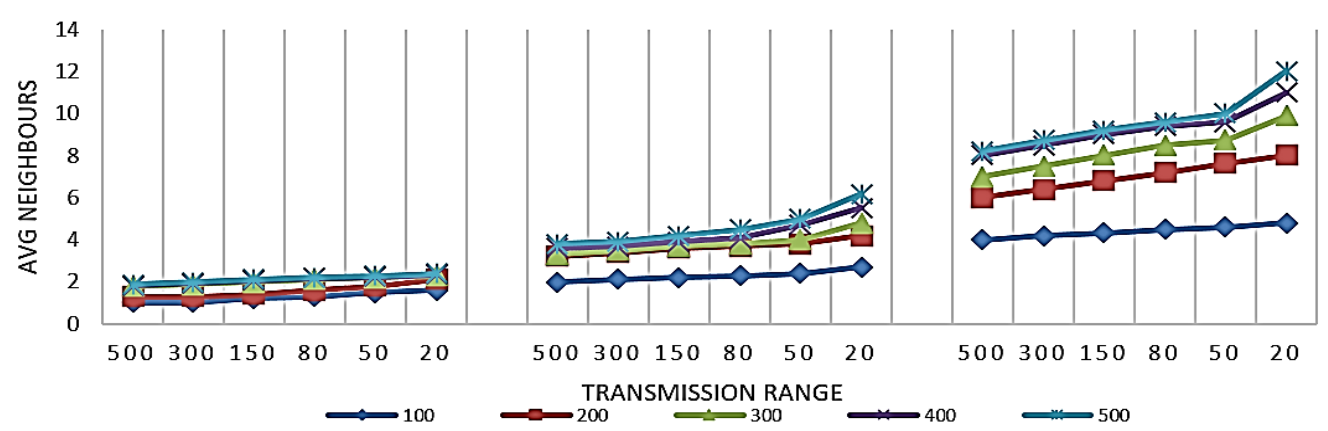

Figure 5. Effect of increasing number of nodes over the average number of neighbors in the asymmetric case

Figure 7 displays the results for the probability of connectivity in the three cases: symmetric links, extended symmetric links and asymmetric links. This figure shows that between the range of $50 \mathrm{~m}$ and $150 \mathrm{~m}$ for the transmission radius (in all three cases), the connectivity experiences a sharp increase. After that point, connectivity tends to be more stabilized. A small increase is observed between $20 \mathrm{~m}$ and $50 \mathrm{~m}$. This figure also shows that the behavior of the three cases is similar in terms of connectivity. To review, in the asymmetric link assumption, connectivity is only affected by the transmission radius. This is also the case with the symmetric and extended symmetric cases. This behavior is consistent for various numbers of mobile nodes.

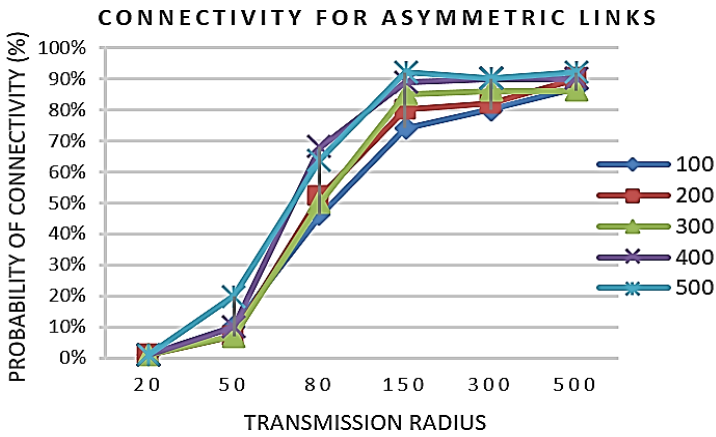

Figure 6. Effect of increasing sensing radius over connectivity in the asymmetric case

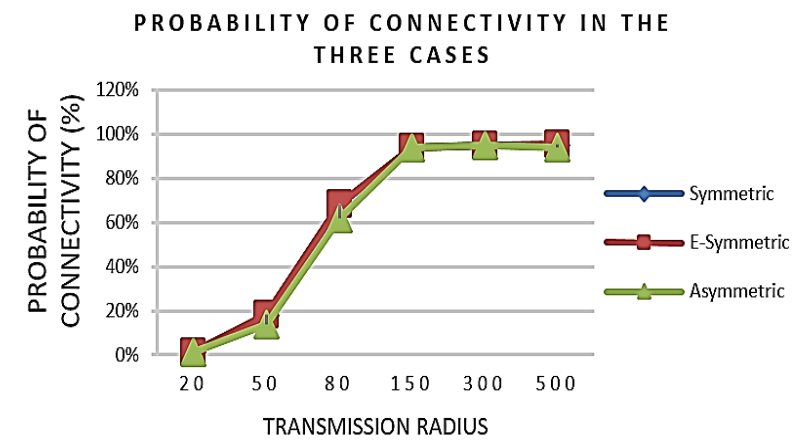

Figure 7. Probability of connectivity in the three cases

Figure 8 presents the effect of increasing sensing and transmitting radii over the average number of nodes for the asymmetric case. The pause time is 0 and number of nodes is 20 . Figure 8 shows the effect of 
increasing the sensing and transmitting radii for mobile nodes over the average number of neighbors for each node in the network for the asymmetric case. This figure supports our observation from the previous sections, i.e., transmission radius has very small effect on the average number of neighbors. Sensing radius on the other hand has a large impact as expected. Figure 9 shows the average number of neighbors in the symmetric, extended symmetric and asymmetric case with pause time 0 . The number of nodes is chosen to be 20 .

Figure 9 displays the results for the average number of neighbors for each node in the three cases: symmetric links, extended symmetric links and asymmetric links. The figure shows that the symmetric and extended symmetric cases behave similarly. Alternatively, in the case of asymmetric links, increasing sensing radius results in a larger average number of neighbors for each node in comparison with the symmetric and extended symmetric cases. We were expecting the values for the three cases to be similar. However, the figure shows that the asymmetric case is a little different than the other two cases. We believe that the effect of transmitting radius on average neighbors as shown in Figures 5 and 8 can explain these results.

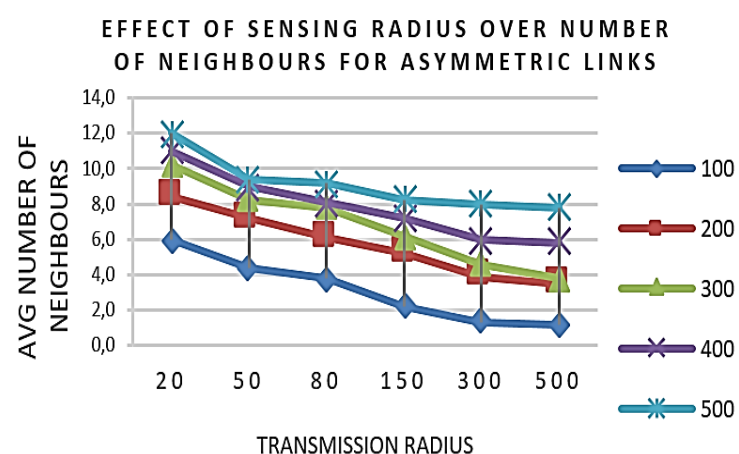

Figure 8. Effect of increasing $T_{r}$ and $T_{s}$ over average number of nodes in the asymmetric case

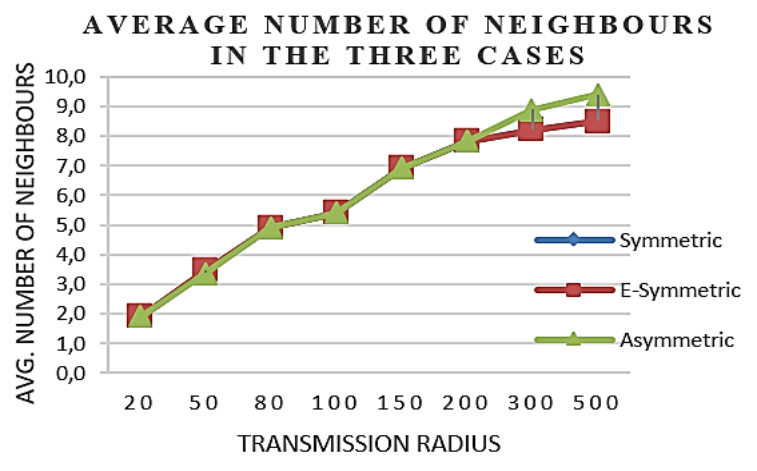

Figure 9. Average number of neighbors in the three cases

\subsection{Effect of mobility results}

Figure 10 presents the effect of varying mean pause time over average number of nodes and connectivity. Figure 10(a) depicts the average number of nodes for mean pause time 0, 10, and 20. Figure 10(b) depicts the results for the probability of connectivity for different pause times. Both the graphs show results for the symmetric and extended symmetric cases with various radii values (first set of lines for symmetric and second set of lines for extended symmetric). Figures 10(a) and 10(b) show that the pause time has a minimal effect on both the average number of nodes and connectivity. Based on this fact, we have shown graphs for pause time 0 alone. We performed analysis for other pause times as well but observed that the results are identical. Therefore, only results for pause time 0 have been included in this report.

Figure 11 depicts the effect of varying pause time over average number of nodes and connectivity in the asymmetric case. Figure 11(a) depicts the average number of nodes for mean pause time 0, 10, and 20. Figure 11(b) depicts the results for the probability of connectivity for different pause times. The first area coverage is for pause time 0 , the second for pause time 10 and the third for pause time 20 in both graphs. This figure also shows that pause time has a minimal effect on both the average number of nodes and connectivity.

\subsection{Symmetric versus asymmetric links results}

Here, we present and discuss the results for the average number of neighbors and connectivity for both the symmetric and asymmetric cases in. Pause time is set to 0 and number of nodes is set to 20 . $T_{r}$ represents the transmission radius and $S_{r}$ represents the sensing radius. In the asymmetric case, for each transmitting radius (e.g. 20), there are 5 values for sensing radius corresponding to 100, 200, 300, 400 and 500 meters. On the other hand, each value for transmitting radius corresponds to one value for sensing radius in the symmetric case. Intuition suggests that the sensing radius is larger than the transmitting radius. Hence, we are concerned about values where this condition holds. Obtained results show that to achieve the same level of connectivity for the asymmetric case as in the symmetric case, the average number of neighbors has increased in the asymmetric case. For example, we can observe that for transmitting radius of $150 \mathrm{~m}$, the average number of neighbors in the symmetric case is 6.8. In case of asymmetric, we have the same level of connectivity but the average numbers of nodes are increased and go up to 9.5 with sensing radius of $500 \mathrm{~m}$. 


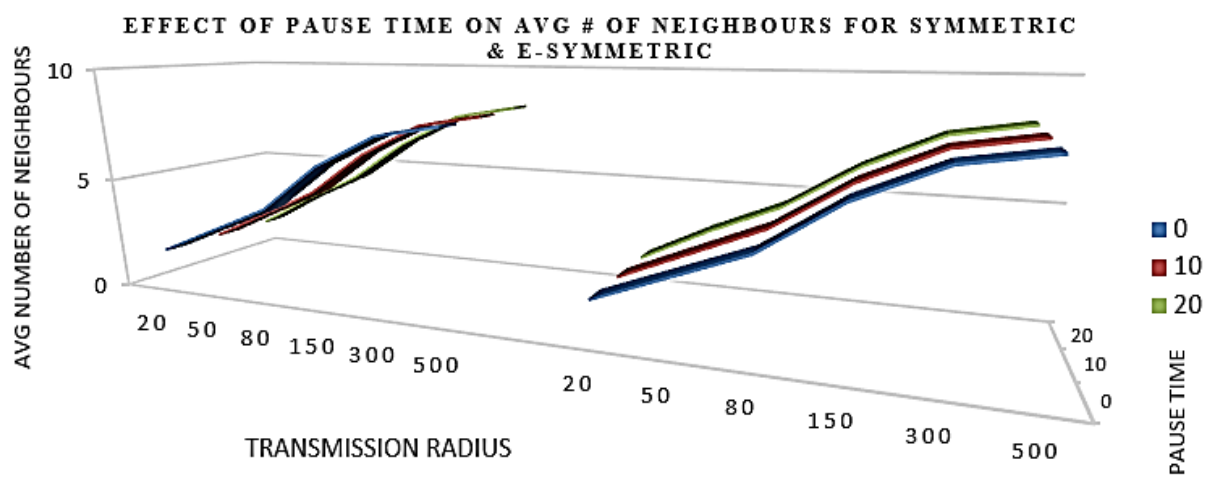

(a)

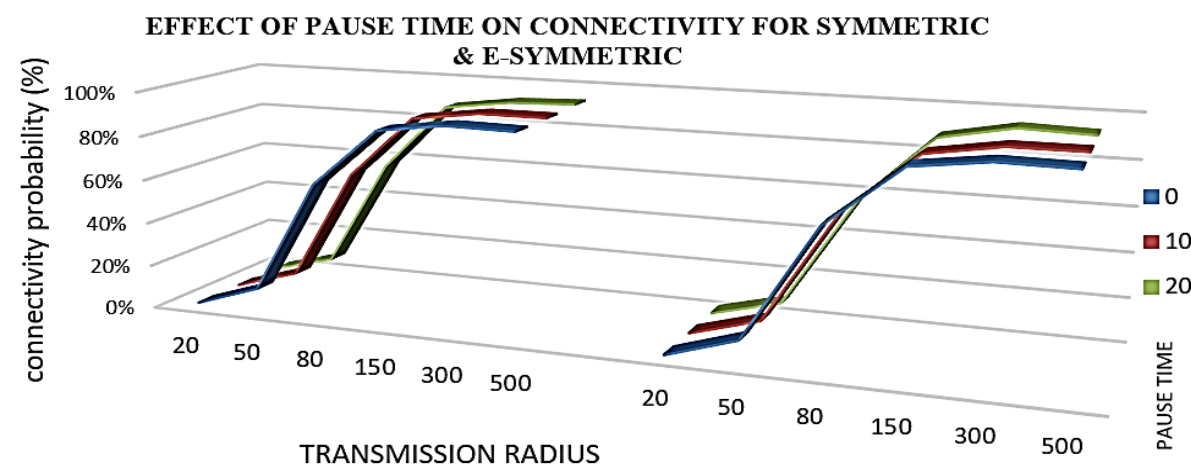

(b)

Figure 10. Effect of pause time over: (a) average number of nodes, (b) probability of connectivity in the symmetric and extended symmetric cases

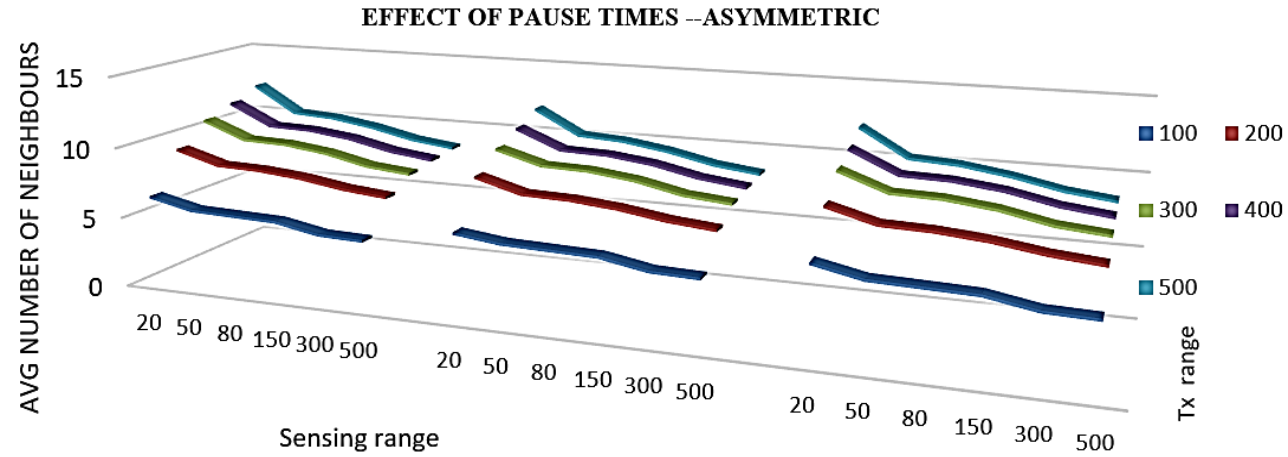

(a)

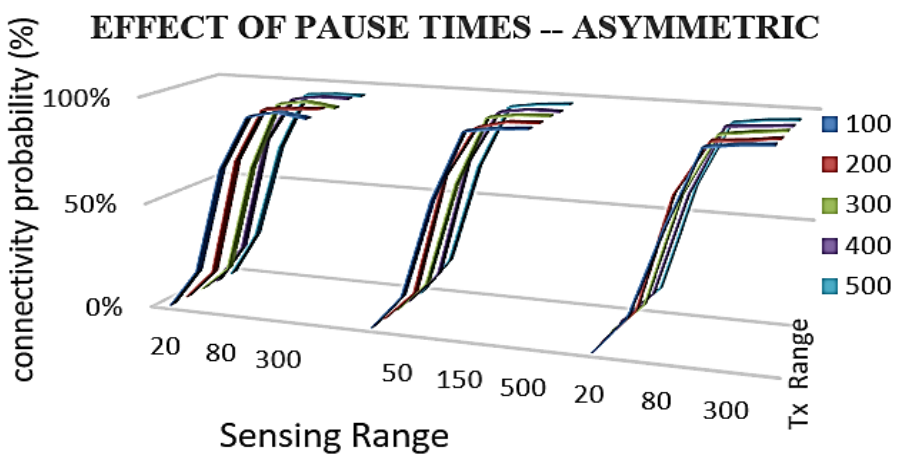

(b)

Figure 11. Effect of pause time over: (a) average number of nodes, (b) probability of connectivity 


\subsection{Effect of environmental factors}

In this part, we investigate the effect of varying the environmental factors on the results for both cases of symmetric and asymmetric assumptions (the case of extended symmetric have a similar performance as the symmetric case). The environmental factors are enormous to be considered individually, hence, we study the aggregate effect of these factors by varying the signal noise ratio (SNR) to reflect the quality of the surrounding environment. High SNR value indicates a less hostile environment. For this, we examine two cases: harsh and soft settings. Figures 12-15 depict the results of both average number of neighbors and connection probability for the soft and harsh environments. The results are depicted for the symmetric and the asymmetric cases. It is clear that the results for the soft environment scenario are much better than the results for the harsh environment scenario. As the transmission radius increases the two indicators increase in both scenarios.

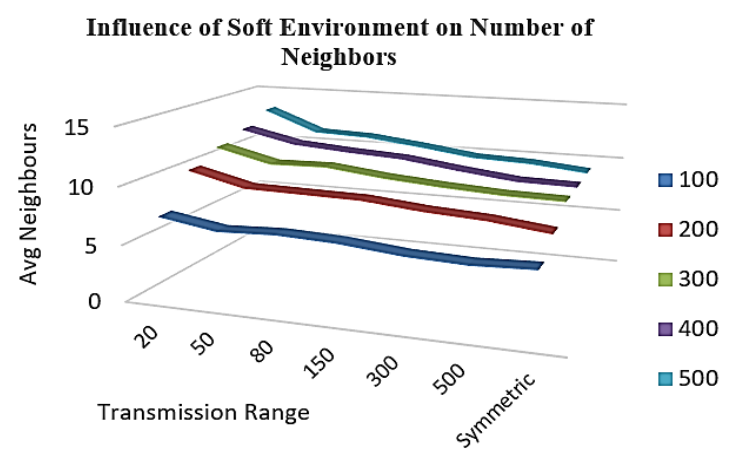

Figure 12. Average number of neighbors-soft environment

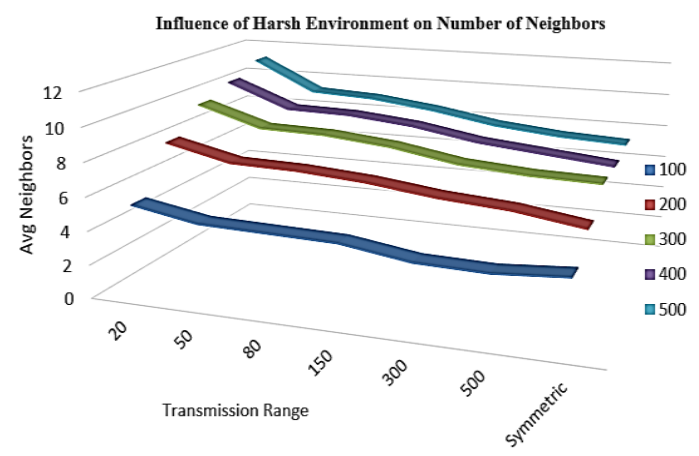

Figure 14. Average \# of neighbors-harsh environment

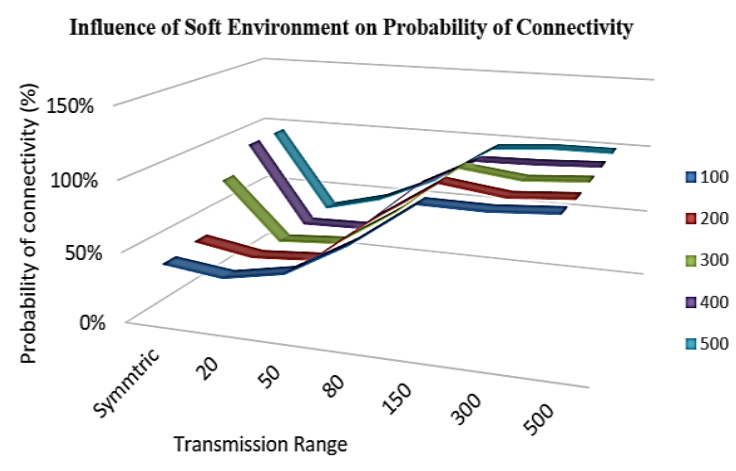

Figure 13. Connection probability-soft environment

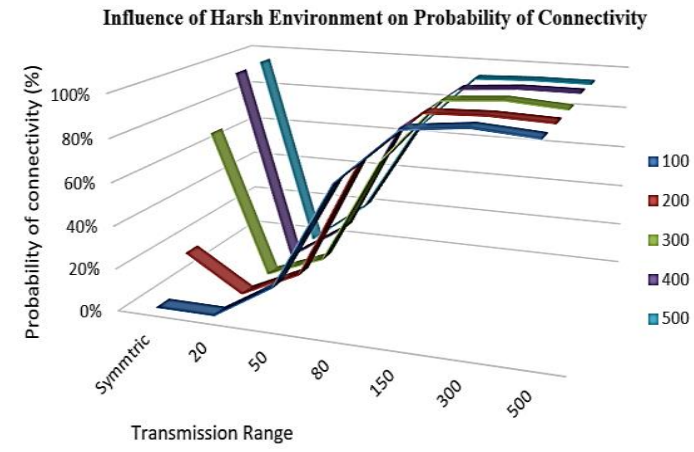

Figure 15. Connection probability-soft environment

\section{RESOURCE ALLOCATION MECHANISM}

The resource allocation problem in wireless networks can be viewed as a scheduling problem with $n$ jobs, each job has a release date $r_{i}$, processing time $p_{i}$, weight $w_{i}$ and deadline $d_{i}$. with $C$ identical machines. Assume the set of jobs is represented by unidirectional graph $G(V, E)$. Graph $\mathrm{G}$ is an interval graph. Each job $j_{i}$ is mapped to a vertex $v_{i}$ in the set $V . j_{i}$ is described by the start time $a_{i}$ and the completion time $b_{i}$. Both $a_{i}$ and $b_{i}$ represent the two end points of interval $I_{i}$. Two vertices are said to be adjacent if and only if the intersection by the corresponding intervals is not null. In this work, we propose an exact and efficient algorithm for both one and two channels problem with the assumptions of fixed rate and delay guarantee. The system supports all users with one of 2 fixed rates. In this framework, each connection can be assigned one of two rates: low rates $(R 1)$ or high rate $(R 2)$, such that $R 1 \leq R 2 \leq 2 R 1$ (i.e., at any time slot the system can either serve one $R 2$ user or two $R l$ users). The system capacity is $C$ channels, here we differentiate between 2 cases: - Case 1: $R 1>R 2=\frac{R 2}{2}$

In this case, the problem can be reduced to the single rate problem by combining two overlapping $R l$ s connections into one $R l$ connection using the following procedure: 
a. For all $R l$ connections

1) If connection $i$ overlaps with connection $j$ then

2) $n e w W[i, j]=w(i)+w(j)$

b. While new $W$ is not empty

1) $\max =\max ($ new $W[i, j])$

2) Create a new connection from connections $i$ and $j$ with release time $r_{\text {new }}=\min \left(r_{i}, r_{j}\right)$, deadline $d_{\text {new }}=\max \left(d_{i}, d_{j}\right)$, and weight $w_{\text {new }}=w(i)+w(j)$

3) delete $n e w W[i, j]$, new $W[*, i]$, new $W[i, *]$, new $W[*, j]$, and $n e w W\left[j,{ }^{*}\right]$.

We propose a 2-channel packing algorithm that accepts either one $R 1$ connection or two $R 2$ connections by packing them together repeated $\frac{C}{2}$ times only. Here is the algorithm:

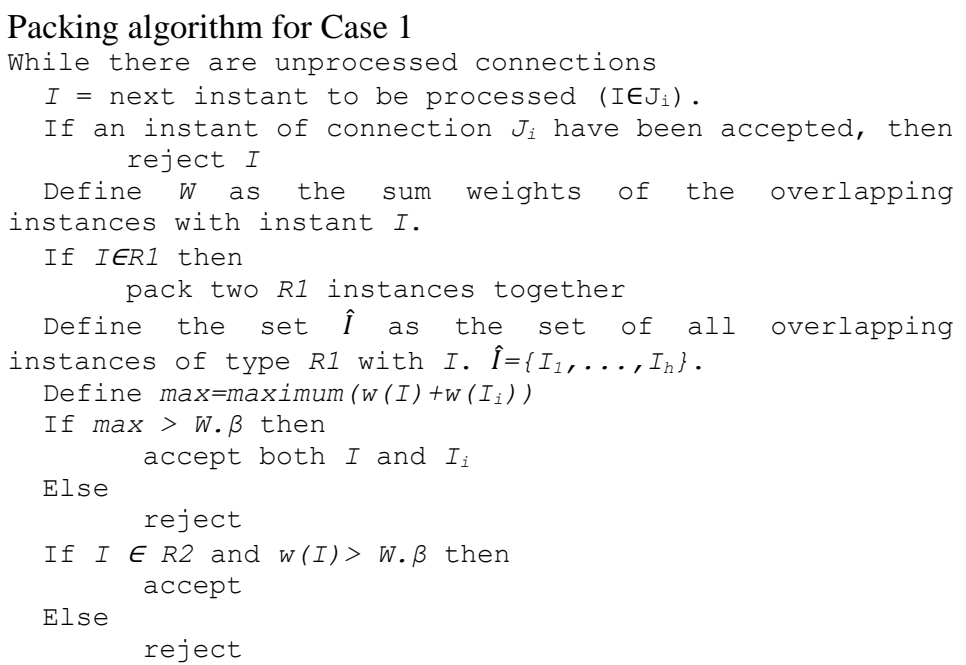

- Case 2: $R 1>R 2>\frac{R 2}{2}$

For now, and for simplicity purposes we assume that each system channel is either equal to $R l$ or $R 2$. In case of it is equal to $R l$ we propose a two-channel packing/scheduling algorithm. Here, we consider connections delay by creating different instances for each connection such that each connection instant starts at time $t \geq r_{i}$ and ends before time $t \leq d_{i}$. At most, one instant of each connection can be scheduled. Now, we propose a greedy algorithm for the dual rate scheduling problem. This algorithm scans all connections from left to right in the timeline (i.e., scans the connections with earlier release date first). The algorithm decided whether to accept or reject an instant. Some accepted connections might get rejected at later stage. As we mentioned before, only one instant of each connection can be accepted. In this case, the use of repetitive method for the multiprocessor case may not result in the maximum weight. This is due to the fact that the two rates are different and $R l$ cannot be reduced to $R 2$ as described in the previous case. We devise the following integer linear program for the dual rate problem. Recall, each connection is described by $\left(r_{i}, p_{i}, d_{i}, w_{i}, c_{i}\right)$, where $c_{i}$ is the connection rate measured in $\mathrm{KB}$. The system total capacity at each time slot is $C$ and is measured by KBs as well. Define the variable $x_{i, t}$ as the number of $\mathrm{KB}$ assigned for user $i$ at time $t$. Time is slotted. Assume that the users' weights are integer values.

$$
\operatorname{Maximize} \sum_{i=1}^{n} \sum_{t=r i}^{d i} w_{i} x_{i t}
$$

Subject to:

For each time slot, $\sum_{i=1}^{n} \sum_{t}^{t+1} x_{i t} \leq C$

For each connection, $\sum_{t=r i}^{d i} x_{i t}=p_{i} c_{i}$

For each connection, $0 \leq x_{i t} \leq c_{i}$

Now, we propose the following algorithm for the dual-rate case. This algorithm runs only for one time and tries to maximize both total connections weight and total use of system's capacity at each time slot. Find-maximum-weight-rate procedure is a greedy one that finds the maximum weight for a given subset of connections taking into account the capacity constraints. 


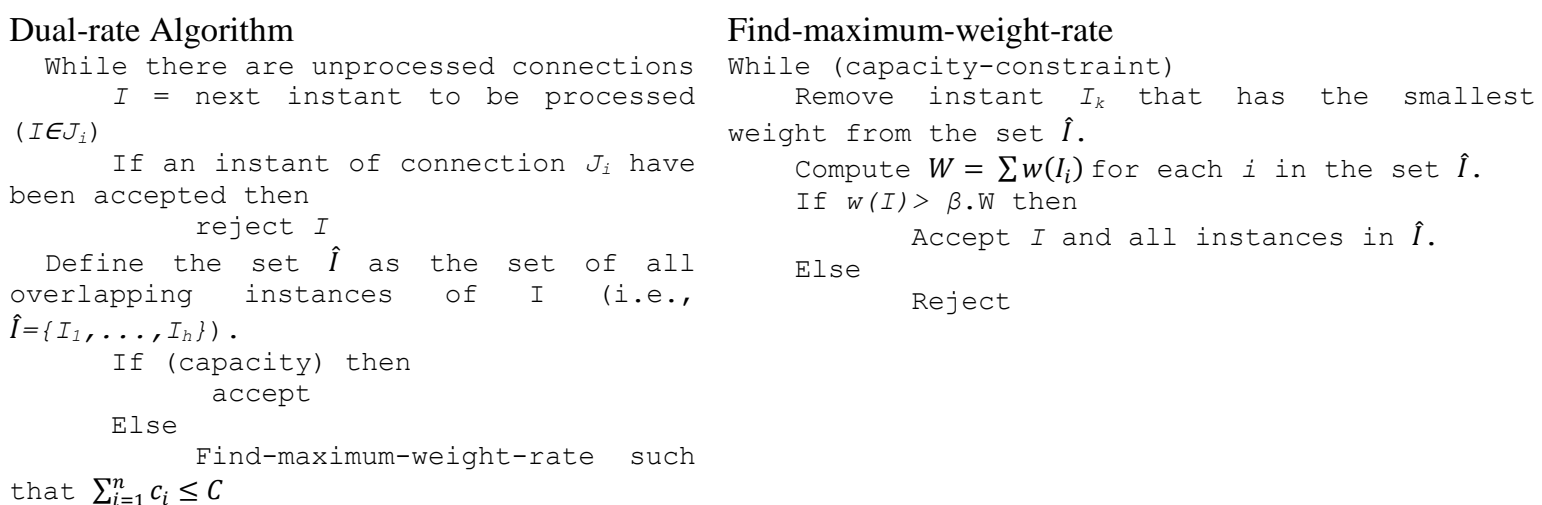

\section{SIMULATION AND RESULTS}

The performance the proposed resource allocation framework is evaluated using simulation, only preliminary results are reported here, a complete analysis of its performance will be conducted in the future work. Resent the simulation environment and obtained results. It is compared again the performance of an early random rejection (ERE) scheme and Genetic algorithm-based scheme [25]. In ERE, connections are accepted up to a certain threshold $\left(T h_{\max }\right)$, once the threshold is reached, new connections are rejected. The system capacity $(C)$ is set to 200 . Network load is expressed by Erlangs, in which Erlang $(E)$ is an expression of both connections arrival rate $(\lambda)$, and the average connection time $(m)$ such that $E=\lambda m$.

To select the best threshold value for the ERE scheme, we conducted several experiments with different $T h_{\max }$ values for the case of 100 Erlangs. The maximal threshold value was achieved at $80 \%$. The remaining results for the ERE scheme was obtained at this threshold value. To measure the performance of these 3 schemes, the network effective throughput and the percentage of successful connections, as well as the delay were used as metrics. Figures 16 to 18 depict these results for these schemes, respectively. We note that the proposed scheme outperformed both ERE and genetic schemes at higher network's loads in terms of throughput and percentage of completed connections and a comparable performance in terms of delay.

EFFECTIVE THROUGHPUT

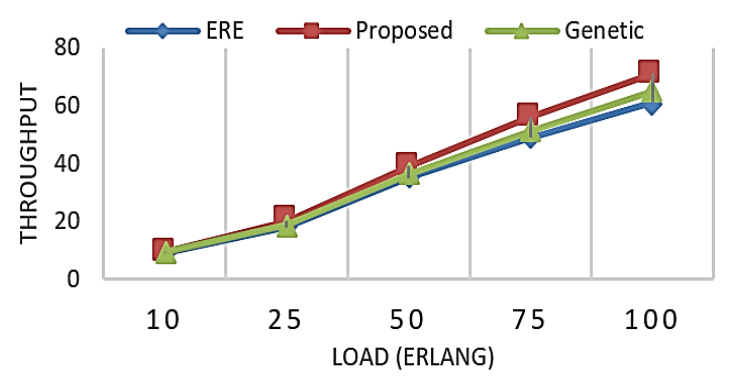

Figure 16. Effective throughput results
FULLY SERVED CONNECTIONS

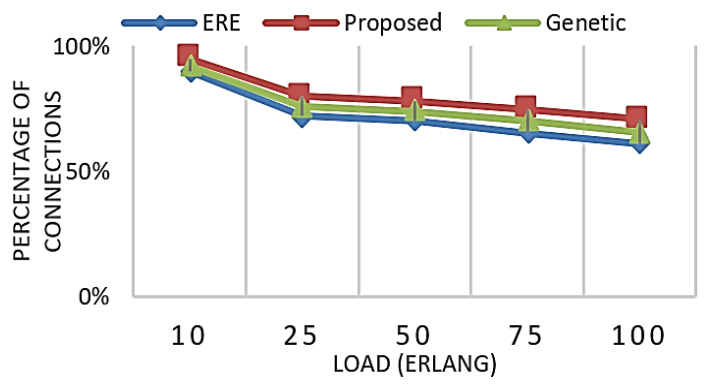

Figure 17. Successfully served connections

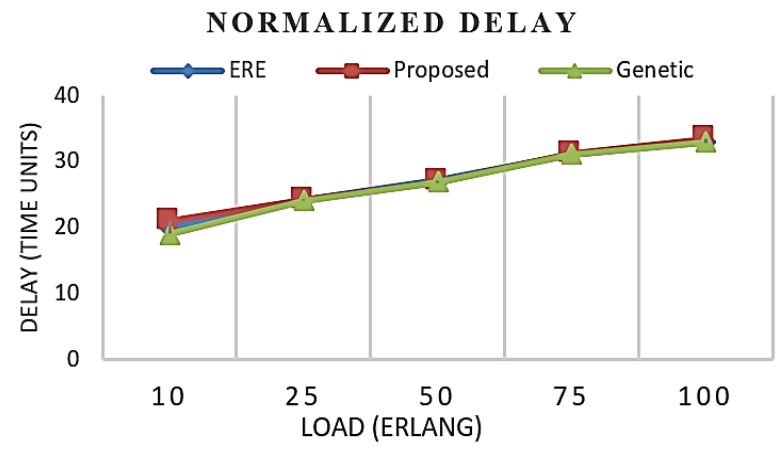

Figure 18. Normalized delay 


\section{CONCLUSION}

Researchers have traditionally held the opinion that mobile networks are symmetric in nature, i.e., mobile nodes have the same transmitting and sensing radius. However, it has been established that this is not always the case. Thus, the goal of this work has been to study the effect of an asymmetric link assumption in mobile networks over the probability of connectivity and the average number of neighbors for each node in the network. In order to pursue with this study, an event-based simulator was developed. The mobility model was based on the popular random waypoint model. The experiments performed in this work have led to the following conclusions, the asymmetric link assumption has a minimal impact on the connectivity of the mobile network. However, the cost (measured in terms of the average number of neighbors for each mobile node in the network) for achieving the same level of connectivity using the asymmetric link assumption increases. Finally, a resource allocation scheme is proposed to serve nodes' connection requests and admits as much as possible of these requests. The performance of this scheme is evaluated using simulation and the obtained preliminary results show that it outperformed the heuristic scheme. This work studied the effect of asymmetric links over connectivity and the cost required to maintain this connectivity. As part of the future work, we will extend the current research idea to cover the effect of this assumption on Interference level (power-noise ratio), routing protocols, and delay. Other future work may include the effect of asymmetric links on other standards such as Bluetooth. Furthermore, the combined effect of the symmetric/asymmetric and the capacity assumption will be further examined.

\section{ACKNOWLEDGMENT}

This work was done in support of the research fund from Jordan University of Science and Technology for sabbatical leave (Fund number 20190220).

\section{REFERENCES}

[1] X. Hu, "Study on wireless local area network technology," 2012 2nd International Conference on Consumer Electronics, Communications and Networks (CECNet), 2012, pp. 609-612, doi: 10.1109/CECNet.2012.6201526.

[2] Y. A. Bangash and Y. E. A. Al-Salhi, "Security issues and challenges in wireless sensor networks: A survey," IAENG International Journal of Computer Science, vol. 44, no. 2, pp. 135-149, 2017.

[3] P. Roshan and J. Leary, 802.11 Wireless LAN Fundamentals, Cisco Press, 2004

[4] N. Golmie, "Interference in the $2.4 \mathrm{GHz}$ ISM band: Challenges and solutions," 2001, Accessed: Jan. 7, 2021. [Online]. Available: https://pdfs.semanticscholar.org/63e2/c826f10b93fecca35745d85aea77831aaab2.pdf

[5] T. S. Rappaport, "Wireless communications: Principles and practice," Prentice Hall communications engineering and emerging technologies series, Upper Saddle River, NJ: Prentice Hall PTR, 2002.

[6] Y. Xiaoqing, Z. Zenglin, and H. Wenting, "Experiment measurements of RSSI for wireless underground sensor network in soil," IAENG International Journal of Computer Science, vol. 45, no. 2, pp. 237-245, 2018.

[7] M. K. Rahim Al-juaifari, H. M. Alshamy, and N. H. A. Khammas, "Power enhancement based link quality for wireless mesh network," International Journal of Electrical and Computer Engineering (IJECE), vol. 11, no. 2, pp. 1388-1394, 2021, doi: 10.11591/ijece.v11i2.pp1388-1394.

[8] J. J. Lei, T. Park, and G. I. Kwon, "A reliable data collection protocol based on erasure-resilient code in asymmetric wireless sensor networks," International Journal of Distributed Sensor Networks, vol. 2013, 2013, doi: 10.1155/2013/730819.

[9] L. Sang, A. Arora, and H. Zhang, "On link asymmetry and one-way estimation in wireless sensor networks," ACM Trans. Sen. Netw., vol. 6, no. 2, pp. 1-25, 2010, doi: 10.1145/1689239.1689242.

[10] P. J. Wan, C. W. Yi, and L. Wang, "Asymptotic critical transmission radius for connectivity in wireless ad hoc networks," IEEE Transactions on Information Theory, vol. 56, no. 6, pp. 2867-2874, Jun. 2010, doi: 10.1109/TIT.2010.2046254.

[11] C. S. Nam, S. T. Bae, J. W. Chung, and D. R. Shin, "Multihop-based optimal cluster heads numbers considering relay node in transmission range of sensor nodes in wireless sensor networks," International Journal of Distributed Sensor Networks, vol. 2013, 2013, doi: 10.1155/2013/480981

[12] W. Raza, N. Javaid, H. Nasir, N. Alrajeh, and N. Guizani, "Buffer-aided relay selection with equal-weight links in cooperative wireless networks," in IEEE Communications Letters, vol. 22, no. 1, pp. 133-136, Jan. 2018, doi: 10.1109/LCOMM.2017.2756833.

[13] H. Audéoud and M. Heusse, "Quick and efficient link quality estimation in wireless sensors networks," 2018 14th Annual Conference on Wireless On-demand Network Systems and Services (WONS), Isola, 2018, pp. 87-90. doi: 10.23919/WONS.2018.8311667.

[14] D. Kotz, C. Newport, and C. Elliott, "The mistaken axioms of wireless-network research," Computer Science Technical Report TR2003-467, 2003.

[15] E. Salahat, A. Kulaib, N. Ali, and R. Shubair, "Exploring symmetry in wireless propagation channels," 2017 European Conference on Networks and Communications (EuCNC), Oulu, 2017, pp. 1-6, doi: 10.1109/EuCNC.2017.7980698.

[16] B. Silva, R. M. Fisher, A. Kumar, and G. P. Hancke, "Experimental link quality characterization of wireless sensor networks for underground monitoring," in IEEE Transactions on Industrial Informatics, vol. 11, no. 5, pp. 1099-1110, Oct. 2015, doi: 10.1109/TII.2015.2471263.

[17] L. Kleinrock and J. Silvester, "Optimum transmission radii for packet radio networks or why six is a magic number," Proceedings of IEEE National Telecommunications Conference, Birmingham, Alabama, Dec. 1978.

[18] E. M. Royer, P. M. M. Smith, and L. E. Moser, "An analysis of the optimum node density for ad hoc mobile networks," IEEE Int. Conference on Communications, vol. 3, 2001, pp. 857-861, doi: 10.1109/ICC.2001.937360.

[19] S. H. Alnabelsi, H. A. B. Salameh, and Z. M. Albataineh, "Dynamic resource allocation for opportunistic software-defined IoT networks: stochastic optimization framework," International Journal of Electrical and Computer Engineering (IJECE), vol 10, no. 4, pp. 3854-3861, 2020, doi: 10.11591/ijece.v10i4.pp3854-3861. 
[20] N. Naderializadeh, J. Sydir, M. Simsek, and H. Nikopour, "Resource management in wireless networks via multi-agent deep reinforcement learning," in IEEE Transactions on Wireless Communications, vol. 20, no. 6, pp. 3507-3523, Jun. 2021, doi: 10.1109/TWC.2021.3051163.

[21] S. Rezwan and W. Choi, "Priority-based joint resource allocation with deep q-learning for heterogeneous NOMA systems," in IEEE Access, vol. 9, pp. 41468-41481, 2021, doi: 10.1109/ACCESS.2021.3065314.

[22] A. Showail, K. Jamshaid, and B. Shihada, "Buffer sizing in wireless networks: challenges, solutions, and opportunities," in IEEE Communications Magazine, vol. 54, no. 4, pp. 130-137, Apr. 2016, doi: 10.1109/MCOM.2016.7452277.

[23] S. R. Das, C. E. Perkins, and E. M. Royer, "Performance comparison of two on-demand routing protocols for ad hoc networks," Proceedings IEEE INFOCOM 2000. Conference on Computer Communications. Nineteenth Annual Joint Conference of the IEEE Computer and Communications Societies (Cat. No.00CH37064), Tel Aviv, Israel, vol. 1, 2000, pp. 3-12, doi: 10.1109/INFCOM.2000.832168.

[24] T. Cormen, C. Leiserson, and R. Rivest, Introduction to algorithms, MIT Press, 1999.

[25] Y. Khamayseh and R. Al-qudah. "Resource allocation using genetic algorithm in multimedia wireless networks," International Journal of Communication Networks and Information Security (IJCNIS), vol. 10, no. 2, pp. 419-424, 2018.

\section{BIOGRAPHIES OF AUTHORS}

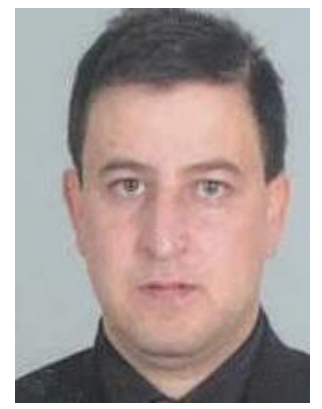

Yaser Khamayseh (iD) $\mathrm{SC}$ SC $\mathrm{P}$ is an associate professor of computer science at Jordan University of Science and Technology. He received his bachelor's degree in computer science from Yarmouk University, Irbid, Jordan, in 1998. He finished his master's in computer science at the University of New Brunswick, Canada in 2001. And his Ph.D. in computer science at University of Alberta, Canada, in 2007. Dr. Khamayseh joined Jordan University of Science and Technology in 2007. Dr. Khamayseh has more than 15 years of experience in research and teaching in the field of data communication and computer networks in different institutions, he worked at TR Labs for testing high speed network devices. His research interests include simulation and modeling, wireless networks, performance evaluation, security, next generation Internet, and evolutionary computation. Dr. Khamayseh has more than 80 published articles in international journals and conferences. Dr. Khamayseh is member of IEEE and has received several awards. He can be contacted at email: yaser@just.edu.jo.

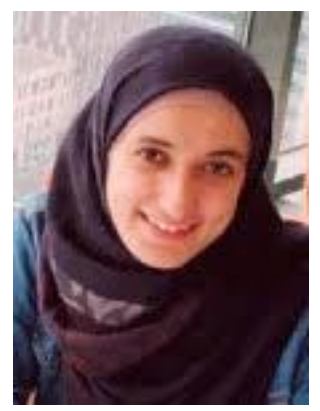

Rabiah Al-Qudah (D) SC P is currently a Ph.D. candidate in computer science at Concordia university. She earned her Msc degree in computer science in 2018 from Concordia university, and her BSc degree in computer science from Jordan University of Science and Technology. She has seven years of experience in software and database development. She published several articles in international conferences and journals. Her research interest includes deep learning and computer aided diagnosis. She was awarded the best paper award in ICVISP 2019. She has seven years of experience in software and database development. She published several articles in international conferences and journals. Her research interest includes deep learning and computer aided diagnosis. She can be contacted at email: r_alquda@encs.concordia.ca. 